\section{Prevalência de hemoglobinas anormais em recém-nascidos da cidade de Natal, Rio Grande do Norte, Brasil}

\author{
Prevalence of abnormal hemoglobins in newborns \\ in Natal, Rio Grande do Norte, Brazil
}

\author{
1 Departamento de \\ Pediatria, Universidade \\ Federal do Rio Grande \\ do Norte, Natal, Brasil. \\ 2 Centro de Hematologia \\ e Hemoterapia do Rio Grande \\ do Norte, Natal, Brasil. \\ 3 Curso de Farmácia, \\ Universidade Federal \\ do Rio Grande do Norte, \\ Natal, Brasil. \\ 4 Departamento de Análises \\ Clínicas e Toxicológicas, \\ Universidade Federal \\ do Rio Grande do Norte, \\ Natal, Brasil. \\ Correspondência \\ Maria Cristina Pignataro \\ Emerenciano de Araújo \\ Departamento de Pediatria \\ Universidade Federal \\ do Rio Grande do Norte. \\ Rua General Cordeiro \\ de Farias s/n, Natal, RN \\ 59010-180, Brasil. \\ cristinapignataro@bol.com.br
}

\begin{abstract}
Hemoglobinopathies are among the most prevalent hereditary diseases in humans. Studies in different areas of Brazil have identified the prevalence of $S$ and C abnormal hemoglobins. The study analyzed 1,940 cord blood samples of newborns from maternity hospitals in $\mathrm{Na}$ tal, Rio Grande do Norte State, to investigate the prevalence of abnormal hemoglobins. All samples were submitted to cellulose acetate electrophoresis using a Tris-EDTA-borate buffer at $p H$ 8.5. Electrophoresis in agar gel pH 6.2 was performed on samples presenting abnormal hemoglobin. Some 37 (1.91\%) of the newborns presented hemoglobinopathies, as follows: 29 (1.50\%) sickle cell trait (Hb FAS), 6 (0.31\%) heterozygous $\mathrm{Hb}$ C (Hb FAC), one (0.05\%) homozygous $\mathrm{Hb} \mathrm{S}$ ( $\mathrm{Hb} \mathrm{FS}$ ), and one (0.05\%) $\mathrm{Hb}$ Barts suggestive of alpha thalassemia. The results show the need to implement screening for hemoglobinopathies in the neonatal population.
\end{abstract}

Sickle Hemoglobin; Sickle Cell Anemia; Neonatal Screening
Maria Cristina Pignataro Emerenciano de Araújo 1

Édvis Santos Soares Serafim 2

Wivel Antonio Pereira de Castro Jr. 3

Tereza Maria Dantas de Medeiros 4

\section{Introdução}

As hemoglobinopatias são doenças geneticamente determinadas e apresentam morbidade significativa em todo o mundo 1 .

Dentre as hemoglobinas anormais, a hemoglobina S é a mais freqüente e os homozigotos ou portadores de anemia falciforme apresentam anemia hemolítica crônica, estando associada a manifestações clínicas importantes desde os primeiros anos de vida, incluindo aumentada suscetibilidade a infecções e recorrentes episódios de oclusão vascular 2,3,4. Embora o traço falciforme (Hb AS) comumente seja assintomático, há relatos de morte súbita e complicações clínicas tais como hematúria, hipostenúria, embolismo pulmonar e infarto esplênico especialmente quando os portadores são expostos a condições extremas de baixa tensão de oxigênio como ocorre em esforços físicos extenuantes, despressurização da cabine de vôo e em grandes altitudes 5 .

A identificação de hemoglobinas anormais em recém-nascidos permite o diagnóstico precoce de portadores de anemia falciforme, evitando ou reduzindo as complicações clínicas da doença, particularmente as infecções pneumocócicas que constituem uma das principais causas de mortalidade nesses pacientes 6,7,8.

Além do mais, a triagem neonatal permite a identificação de portadores e de casais de risco pelo estudo retrospectivo das famílias 
dos recém-nascidos afetados, às quais poderá ser dado aconselhamento genético que possibilite uma decisão consciente em futura gravidez 9 .

As duas hemoglobinas anormais mais freqüentes na população brasileira são as hemoglobinas $\mathrm{S}$ e $\mathrm{C}$, ambas de origem africana, evidenciando a intensa participação do negro africano na composição populacional brasileira 10,11. Esse fato é bem caracterizado nos estudos de prevalência de hemoglobinopatias realizados em diferentes regiões do Brasil 12,13,14,15,16, $17,18,19,20,21$.

Nesse estudo objetivamos investigar a prevalência de hemoglobinopatias em neonatos por intermédio da triagem em sangue de cordão umbilical em uma população da cidade de Natal, Rio Grande do Norte, Brasil.

\section{Casuística e métodos}

No período de janeiro a dezembro de 2001, foram analisadas 1.940 amostras de sangue de cordão umbilical de recém-nascidos provenientes de três maternidades públicas da cidade de Natal: 830 amostras da Maternidade Escola Januário Cicco (MEJC), da Universidade Federal do Rio Grande do Norte (UFRN); 797 do Hospital e Maternidade Santa Catarina, da Secretaria Estadual de Saúde; e 313 da Unidade Mista Materno-Infantil de Lagoa Seca, da Secretaria Municipal de Saúde do Estado do Rio Grande do Norte.

Os recém-nascidos foram escolhidos aleatoriamente, independentemente da idade gestacional e tipo de parto, após as mães serem informadas sobre o estudo e assinarem o Termo de Consentimento Consciente. As variáveis avaliadas durante a coleta foram grupo étnico materno, naturalidade materna e procedência das amostras. O grupo étnico materno foi definido por branco, pardo e negro, considerando critérios como cor da pele, textura do cabelo e formato do nariz e lábios.

A coleta do sangue foi efetuada pelo obstetra responsável pelo parto após o clampeamento do cordão umbilical, colocadas em tubos contendo anticoagulante (EDTA) na proporção de $1 \mathrm{mg} / \mathrm{mL}$ de sangue e acondicionadas em geladeira até o momento das análises, não excedendo 48 horas. Inicialmente, os eritrócitos foram lavados três vezes em solução de cloreto de sódio 0,154 M a 20॰C e, em seguida, lisados com saponina a $1 \% 10$. Todas as amostras foram submetidas à eletroforese de hemoglobina em acetato de celulose utilizando-se tampão TrisEDTA-Borato pH 8,5 22. As amostras que apre- sentaram hemoglobinas anormais em $\mathrm{pH}$ alcalino foram submetidas à eletroforese em gel de ágar pH 6,2, para confirmação das hemoglobinas anormais $\mathrm{S}$ e $\mathrm{C} 23$.

Foi realizado estudo familiar de dez recém-nascidos que apresentaram hemoglobinas anormais e retornaram após os dois meses de vida para confirmação do diagnóstico. As amostras de sangue dos pais e das crianças foram submetidas à eletroforese em pH alcalino, e aquelas que apresentaram hemoglobinas anormais foram confirmadas mediante eletroforese de hemoglobina em $\mathrm{pH}$ ácido e teste de solubilidade 24 .

A análise estatística dos dados foi baseada em cálculos percentuais das variáveis qualitativas. O teste do qui-quadrado $\left(\chi^{2}\right)$ foi utilizado com a finalidade de comparar as freqüências, utilizando-se nível de significância de 5\%. A parte computacional foi realizada com base no pacote estatístico Statistica for Windows by StatSoft, inc. versão 5.1, edição 1997.

O projeto de pesquisa foi previamente aprovado pelo Comitê de Ética em Pesquisa da UFRN e desenvolvido cumprindo integralmente os princípios éticos estabelecidos na Resolução 196/96 do Conselho Nacional de Saúde.

\section{Resultados}

A Tabela 1 apresenta os tipos de hemoglobinas encontradas nas análises das 1.940 amostras de sangue de cordão umbilical, relacionando os seus genótipos com o grupo étnico materno. Do total analisado, $1.903(98,09 \%)$ crianças apresentaram perfil eletroforético normal FA. $\mathrm{O}$ traço falciforme (genótipo FAS) foi o mais prevalente, representando $1,5 \%$, e a anemia falciforme (genótipo FS) correspondeu a $0,05 \%$. Foram ainda detectados os genótipos FAC $(0,31 \%)$ e a hemoglobina FA+Bart's $(0,05 \%)$.

As freqüências gênicas verificadas foram 0,9905 para o gene $\beta \mathrm{A}, 0,0080$ (gene $\beta^{\mathrm{S}}$ ) e 0,0015 para o gene $\beta C$. Valendo-se do cálculo de HardyWeinberg, observou-se por meio do teste quiquadrado $(\mathrm{p}<1)$ que a distribuição dos fenótipos na população se encontra dentro do esperado.

Na Tabela 2, são apresentadas as prevalências das hemoglobinas anormais dos recémnascidos conforme a naturalidade materna. Do total das 1.940 amostras, 37 apresentaram hemoglobinas anormais, representando uma prevalência de 1,91\%. A maior prevalência de hemoglobinas anormais $(2,11 \%)$ foi encontrada nas crianças cujas mães eram provenientes de outros estados do Brasil, porém residentes na 
Prevalência de hemoglobinas anormais em recém-nascidos conforme o grupo étnico materno.

\begin{tabular}{|c|c|c|c|c|c|c|c|c|c|c|c|c|}
\hline \multirow{2}{*}{\multicolumn{3}{|c|}{$\begin{array}{l}\text { Grupo étnico } \\
\text { materno }\end{array}$}} & \multicolumn{2}{|c|}{ FAS } & \multicolumn{2}{|c|}{$\begin{array}{c}\text { Genótipos } \\
\text { FAC }\end{array}$} & \multicolumn{2}{|c|}{ FS } & \multicolumn{2}{|c|}{$\mathrm{FA}+$ Bart's } & \multicolumn{2}{|c|}{ Total } \\
\hline & & $\%$ & $\mathrm{n}$ & $\%$ & $\mathrm{n}$ & $\%$ & $\mathrm{n}$ & $\%$ & $\mathrm{n}$ & $\%$ & $n$ & $\%$ \\
\hline Branco & 519 & 26,75 & 7 & 0,36 & 0 & 0,00 & 0 & 0,00 & 0 & 0,00 & 526 & 27,10 \\
\hline Pardo & 1.179 & 60,77 & 21 & 1,09 & 4 & 0,21 & 1 & 0,05 & 0 & 0,00 & 1.205 & 62,10 \\
\hline Negro & 205 & 10,57 & 1 & 0,05 & 2 & 0,10 & 0 & 0,00 & 1 & 0,05 & 209 & 10,80 \\
\hline Total & 1.903 & 98,09 & 29 & 1,50 & 6 & 0,31 & 1 & 0,05 & 1 & 0,05 & 1.940 & 100,00 \\
\hline
\end{tabular}

$\chi^{2}(2)=1,34$

$\mathrm{p}=0,49$.

FAS = Genótipo normal; FAZ = Traço falciforme; FAC = Traço para hemoglobina $C ; F S=$ Homozigoto

para hemoglobina S (anemia falciforme); FA+Bart's = Heterozigoto para alfa talassemia.

Prevalência de hemoglobinas anormais em recém-nascidos conforme a naturalidade materna.

\begin{tabular}{|c|c|c|c|c|c|c|c|c|c|c|c|}
\hline \multirow[t]{2}{*}{$\begin{array}{l}\text { Naturalidade } \\
\text { materna }\end{array}$} & \multirow{2}{*}{$\begin{array}{l}\text { Número } \\
\text { de amostras } \\
\text { testadas }\end{array}$} & \multicolumn{2}{|c|}{ FAS } & \multicolumn{2}{|c|}{$\begin{array}{c}\text { Genótipos } \\
\text { FAC }\end{array}$} & \multicolumn{2}{|c|}{ FS } & \multicolumn{2}{|c|}{$\mathrm{FA}+$ Bart's } & \multicolumn{2}{|c|}{ Total } \\
\hline & & $\mathrm{n}$ & $\%$ & $\mathrm{n}$ & $\%$ & $\mathrm{n}$ & $\%$ & $\mathrm{n}$ & $\%$ & $\mathrm{n}$ & $\%$ \\
\hline I & 1.110 & 21 & 1,89 & 2 & 0,18 & 0 & 0,00 & 0 & 0,00 & 23 & 2,07 \\
\hline II & 688 & 7 & 1,02 & 2 & 0,29 & 1 & 0,15 & 1 & 0,15 & 11 & 1,60 \\
\hline III & 142 & 1 & 0,70 & 2 & 1,41 & 0 & 0,00 & 0 & 0,00 & 3 & 2,11 \\
\hline Total & 1.940 & 29 & 1,50 & 6 & 0,31 & 1 & 0,05 & 1 & 0,05 & 37 & 1,91 \\
\hline
\end{tabular}

$\chi^{2}(2)=0,53$

$\mathrm{p}=0,77$

I = Grande Natal (Natal, Parnamirim, Ceará-Mirim, São Gonçalo do Amarante, Macaíba e Extremoz);

II = Interior do Estado do Rio Grande do Norte; III = Outros Estados do Brasil.

Cidade de Natal, com prevalência de 0,70\% para o traço falciforme e $1,41 \%$ para o traço da hemoglobina C. Tomando-se por base as 1.798 amostras de crianças cujas mães eram naturais do Estado do Rio Grande do Norte (Grande Natal e interior do Estado), 34 apresentaram hemoglobinas anormais, representando uma prevalência de 1,89\%.

Os dados expostos na Tabela 3 mostram os genótipos das dez crianças portadoras de hemoglobinas anormais, que retornaram para confirmação, e de seus familiares.

\section{Discussão}

A cidade de Natal, capital do Estado do Rio Grande do Norte está situada na Região Nordeste do Brasil e tem sua composição étnica resultante da miscigenação de índios, brancos e negros africanos. A maioria dos brancos veio de Portugal; os negros, principalmente da Costa Oeste da África; e os índios das tribos Potiguar (Tupi), Tapuia e Cariri 25,26.

Atualmente, de acordo com os resultados obtidos na Pesquisa Nacional por Amostra de Domicílio, o Rio Grande do Norte tem um total de 2.777.509 habitantes. Desse total, 1.454.665 (52,37\%) foram classificados como pardos, $1.171 .699(42,19 \%)$ brancos, 126.441 (4,55\%) negros, $2.374(0,09 \%)$ da raça amarela, 3.169 $(0,11 \%)$ de origem indígena e $19.161(0,69 \%)$ sem declaração 27.

A amostra estudada foi constituída por uma maior percentagem de recém-nascidos cujas mães eram pardas, representando $62,10 \%$ da amostra. O grupo étnico branco contribuiu com $27,10 \%$ e o negro com $10,80 \%$ (Tabela 1 ). As discrepâncias entre os percentuais da amostra analisada e os dados do IBGE se dão em virtude da dificuldade de caracterização do grupo étnico pela cor da pele. 
Tabela 3

Resultados dos genótipos das crianças portadoras de hemoglobinas anormais e seus familiares, após confirmação.

\begin{tabular}{lcccc}
\hline \multirow{2}{*}{ Identificação } & \multicolumn{4}{c}{ Genótipos } \\
& Criança & Pai & Mãe & Irmão \\
\hline 1 (E. R. T.) & AS & AA & AS & AA \\
2 (F. L. C. O.) & AS & AS & AS & - \\
4 (M. F. A. S.) & AS & AS & AA & - \\
7 (G. N. A.) & SF & AS & AS & - \\
9 (A. D. N.) & AS & AS & AA & - \\
16 (G. P. C.) & AS & AS & AA & - \\
18 (A. C. M. L.) & AS & AS & AA & - \\
24 (E. C. L. A.) & AC & AC & AA & - \\
31 (T. S. N.) & AS & AA & AS & - \\
33 (A. L. S.) & AS & AS & AA & - \\
\hline
\end{tabular}

A prevalência das hemoglobinas S e C encontradas nesse estudo não difere dos resultados obtidos em estudo anterior realizado na população de Natal como também de outros autores que analisaram essa distribuição em diversas regiões do Brasil 11,13,16,17,19,20,28.

Analisando-se a prevalência de hemoglobinas anormais de acordo com a naturalidade materna foi encontrado um maior percentual nas crianças cujas mães eram provenientes de outros estados do Brasil, contudo, residentes na cidade de Natal, representando $2,11 \%$ com prevalência de $0,70 \%$, para o traço falciforme, e $1,41 \%$, para o traço da hemoglobina C (Tabela 2). Entretanto, não houve diferença estatisticamente significativa entre os grupos $(p=0,07)$.

A cromatografia líquida de alta resolução (HPLC) é considerada uma técnica altamente sensível para o estudo das hemoglobinas anormais porque evidencia pequenas quantidades de $\mathrm{Hb} \mathrm{A}$, bem como $\mathrm{Hb} \mathrm{S}, \mathrm{Hb} \mathrm{C}$ e outras hemoglobinas anormais, na presença de grandes quantidades de $\mathrm{Hb}$ F. Os sistemas automatizados proporcionam um processamento rápido das amostras com um alto índice de reprodutibilidade e exatidão, constituindo-se num excelente método para triagem neonatal 6 . Todavia, na literatura, vários trabalhos demonstram a eficácia da eletroforese de hemoglobina em $\mathrm{pH}$ alcalino, seguida de eletroforese em $\mathrm{pH}$ ácido para a detecção de hemoglobinopatias em sangue de cordão 8,12,15,29.

A metodologia empregada nesse estudo mostrou-se sensível para diagnosticar hemoglobinas anormais em sangue de cordão. Obteve-se boa separação das hemoglobinas S e C e das hemoglobinas A e F, além da detecção de hemoglobina Bart's, encontrada em 0,05\% da amostra total analisada, o que sugere o diagnóstico de portador de alfa talassemia.

A presença da hemoglobina Barts também foi observada por Ruiz et al. 28, utilizando gel de ágar de amido, encontrando uma prevalência de $0,39 \%$ ao analisar 2.281 amostras de sangue de cordão umbilical na cidade de Santos, São Paulo. Um estudo mais recente realizado por Viana-Baracioli et al. 20, utilizando eletroforese em $\mathrm{pH}$ alcalino e pesquisa de hemoglobina $\mathrm{H}$ intra-eritrocitária, evidenciou a prevalência de 4,38 \% de $\mathrm{Hb}$ Barts em amostras de sangue de cordão umbilical de recém-nascidos do Hospital de Base de São José do Rio Preto, São Paulo.

$\mathrm{Na}$ literatura, existem várias publicações relacionadas ao diagnóstico neonatal das hemoglobinopatias em diversos países, demonstrando a importância desse procedimento na redução da mortalidade em crianças menores de cinco anos de idade 29,30,31,32.

No Brasil, diversos pesquisadores têm realizado estudos para a identificação de hemoglobinas anormais em recém-nascidos. Ramalho et al. 33 pesquisaram a hemoglobina $S$ em sangue de cordão umbilical de 220 recém-nascidos na cidade de Campinas, São Paulo, encontrando a prevalência de $2,72 \%$ para o traço falciforme. A mesma prevalência para o heterozigoto da hemoglobina S foi encontrada por Ruiz et al. 28 , em um estudo realizado com 2.281 recém-nascidos da cidade de Santos. No Estado de Minas Gerais, o programa de triagem neonatal para doença falciforme iniciado em março de 1998, analisou 605.419 crianças, encontrando uma prevalência de $3,2 \%$ para o traço falciforme, 1,3\% para o portador da hemoglobina C e $0,08 \%$ para doenças falciformes 34 . Recentemente, estudos realizados por Ducatti et al. 15, em São José do Rio Preto, São Paulo, e Daudt et al. 14, em Porto Alegre, Rio Grande do Sul, evidenciaram uma prevalência de $3,72 \%$ e 1,2 $\%$, respectivamente, para o traço falciforme.

A prevalência de $1,5 \%$ para o traço falciforme encontrada nesse estudo mostrou-se inferior à observada por Bandeira et al. 12 em 1.988 recém-nascidos de Pernambuco. Essa diferença pode ser explicada pelo grande contingente de escravos africanos trazidos para aquele estado durante o período colonial brasileiro, fato que não ocorreu no Estado do Rio Grande do Norte.

Foram enviadas correspondências a todas as mães dos recém-nascidos que apresentaram hemoglobinas anormais com o intuito de convidá-las a comparecer ao ambulatório de hematologia do Hemonorte, para receberem o aconselhamento genético. 
Das 37 crianças portadoras de hemoglobinopatias detectadas, apenas dez retornaram, após dois meses, para confirmação do diagnóstico e realização do estudo familiar (Tabela 3). Admite-se que o retorno foi prejudicado pela dificuldade de localização de endereço de algumas mães, pela falta de conhecimento suficiente sobre a importância do diagnóstico dessa alteração genética e pelo reduzido poder aquisitivo da maioria delas.

Foram detectados dois casais considerados de risco, heterozigotos para a hemoglobina $S$ (Tabela 3). Os pais da criança, a qual apresentou traço falciforme, após receberem orientação médica sobre a alteração genética e a probabilidade do nascimento de filhos com anemia falciforme, decidiram não mais arriscar uma nova gestação. A criança diagnosticada como portadora de anemia falciforme foi inscrita no ambulatório de hematologia do Hemonorte para acompanhamento médico, incluindo o uso de terapia preventiva com antibiótico e esquema de vacinação.

A Portaria do Ministério da Saúde no 822, de 6 de junho de 2001, publicada no Diário Ofi-

\section{Resumo}

As hemoglobinopatias estão incluídas dentre as doenças hereditárias mais freqüentes nas populações humanas. Estudos realizados em diferentes regiões do Brasil têm demonstrado que as hemoglobinas anormais $S$ e C são as mais prevalentes. Com o objetivo de investigar a prevalência de hemoglobinas anormais no período neonatal, foram analisadas 1.940 amostras de sangue de cordão umbilical provenientes de recém-nascidos de três maternidades da cidade de $\mathrm{Na}$ tal, Rio Grande do Norte. Todas as amostras foram submetidas à eletroforese de hemoglobina em acetato de celulose utilizando tampão Tris-EDTA-Borato $\mathrm{pH}$ 8,5. As amostras que apresentaram hemoglobinas anormais foram submetidas à eletroforese em gel de ágar pH 6,2 para confirmação. Foram identificadas 37 (1,91\%) amostras com hemoglobinas anormais, das quais 29 (1,50\%) com traço falciforme (Hb FAS), 06 $(0,31 \%)$ com Hb C, uma (0,05\%) com anemia falciforme (Hb FS) e uma (0,05\%) apresentou Hb Bart's,sugerindo alfa talassemia. Os resultados encontrados evidenciam a necessidade de implantação da triagem de hemoglobinopatias em recém-nascidos na nossa população.

Hemoglobina Falciforme; Anemia Falciforme; Triagem Neonatal cial da União de 7 de junho de 2001, determinou a obrigatoriedade do Programa Nacional de Triagem Neonatal em todo o Território Nacional, tendo como objetivo o desenvolvimento de ações de triagem neonatal, diagnóstico, acompanhamento e tratamento das doenças congênitas como fenilcetonúria, hipotireoidismo congênito, fibrose cística, doenças falciformes e outras hemoglobinopatias 35 .

Levando-se em conta a extensão de nosso país, a miscigenação racial e a prevalência das hemoglobinopatias, torna-se necessária a expansão dos centros de triagem para detectar estas alterações genéticas, a fim de permitir um melhor conhecimento sobre esse grupo de doenças hereditárias.

No Estado do Rio Grande do Norte não há relato de estudos referentes à identificação de hemoglobinas anormais no período neonatal. Os resultados obtidos nesse estudo permitem recomendar a implementação de um programa para triagem neonatal de hemoglobinopatias em nosso Estado, oferecendo orientações e aconselhamento genético às famílias das crianças portadoras de hemoglobinas anormais.

\section{Colaboradores}

M. C. P. E. Araújo contribuiu na elaboração do projeto de pesquisa, coleta de amostras, análises laboratoriais, interpretação dos dados e redação do texto. W. A. P. Castro Jr. auxiliou na execução das análises laboratoriais. E. S. S. Serafim participou do acompanhamento e orientação médica aos pais e familiares dos recém-nascidos que apresentaram hemoglobinas anormais. T. M. D. Medeiros contribuiu na execução das análises laboratoriais e redação do texto, assim como na revisão final da versão para publicação. 


\section{Referências}

1. Weatherall DJ, Clegg JB. Genetic disorders of hemoglobin. Semin Hematol 1999; 36:24-37.

2. Leiken SL, Gallagher D, Kinney TR. Mortality in children and adolescents with sickle cell disease. Pediatrics 1989; 84:500-8.

3. Powars D. Diagnosis at birth improves survival of children with sickle cell anemia. Pediatrics 1989; 83:830-3.

4. Rodgers DW, Clarke JM, Cupidore L. Early deaths in Jamaica children with sickle cell disease. $\mathrm{Br}$ Med J 1978; 1:1515-16.

5. Harkness DR. Sickle cell trait revisited. Am J Medicine 1989; $87: 30 \mathrm{~N}-34 \mathrm{~N}$

6. Chapman CS. Neonatal screening for haemoglobinophaties. Clin Lab Haematol 1999; 21:229-34.

7. Gaston MH, Verter JI, Woods G, Pegelow C, Kelleher J, Presbury G, et al. Prophylaxis with oral penicillin in children with sickle cell anemia: a randomized trial. N Engl J Med 1986; 314:1593-99.

8. Githens JH, Lane PA, McCurdy RS, Houston ML McKinna JD, Cole DM. Newborn screening for hemoglobinopathies in Colorado. Am J Dis Child 1990; 144:466-70.

9. Peres MJ, Carneiro MH, Machado MC, Seixas T, Picanço I, Batalha L, et al. Rastreio neonatal de hemoglobinopatias numa população residente em Portugal. Acta Med Port 1996; 9:135-9.

10. Naoum PC. Hemoglobinopatias e talassemias. São Paulo: Editora Sarvier; 1997.

11. Salzano FM, Tondo CV. Hemoglobin types in Brazilian populations. Hemoglobin 1982; 6:85.

12. Bandeira FMGC, Leal MC, Souza RR, Furtado VC, Gomes YM, Marques NM. Características de recém-nascidos portadores de hemoglobinas "S" detectados através de triagem em sangue de cordão umbilical. J Pediatr (Rio J.) 1999; 75:167-71.

13. Bezerra TMM; Albuquerque LMM, Lima AAB, Andrade SR. Prevalência de hemoglobinas anormais em Natal, Rio Grande do Norte. Rev Bras Anal Clin 1991; 23:73-6.

14. Daudt LE, Zechmaister D, Portal L, Camargo Neto E, Silla LMR, Giugliani R. Triagem neonatal para hemoglobinopatias: um estudo piloto em Porto Alegre, Rio Grande do Sul, Brasil. Cad Saúde Pública 2002; 18:833-41.

15. Ducatti RP, Teixeira AEA, Galão HA, Bonini-Domingos CR, Fett-Conte AC. Investigação de hemoglobinopatias em sangue de cordão umbilical de recém-nascidos do Hospital de Base de São José do Rio Preto. Rev Bras Hematol Hemoter 2001; 23:23-9.

16. Moreira HW, Monteiro SCM, Marques TC, Oliveira FS, Bertholo LC, Gimenez I. Triagem das hemoglobinopatias por isoeletrofocalização. Jornal Brasileiro de Patologia 1999; 35:141-5.

17. Naoum PC, Alvares Filho F, Domingos CRB, Ferrari F, Moreira HW, Sampaio ZA, et al. Hemoglobinas anormais no Brasil. Prevalência e distribuição geográfica. Revista Brasileira de Patologia Clínica 1987; 23:68-79.

18. Orlando GM, Naoum PC, Siqueira FAM, BoniniDomingos CR. Diagnóstico laboratorial de hemoglobinopatias em populações diferenciadas. Rev Bras Hematol Hemoter 2000; 22:111-21.
19. Tavares Neto J, Naoun PC, Adorna J, Azevêdo P, Brito F, Caldas M, et al. Hemoglobinopatias no Distrito Federal, Brasil. Rev Soc Bras Med Trop 1986; 19:13-9

20. Viana-Baracioli LMS, Bonini-Domingos CR, Pagliusi RA, Naoum PC. Prevenção de hemoglobinopatias a partir do estudo em gestantes. Rev Bras Hematol Hemoter 2001; 23:31-9.

21. Zago MA, Costa FF, Tone LG, Botura C. Hereditary hemoglobins disorders in Brazilian population. Hum Hered 1983; 33:125-9.

22. Marengo-Rowe AJ. Rapid electrophoresis and quantification of hemoglobin on cellulose acetate. J Clin Pathol 1965; 18:790-2.

23. Vella F. Acid agar gel electrophoresis of human hemoglobins. Am J Clin Pathol 1968; 49:440.

24. Magalhães E, Arashiro DN. Um método simples para visualização da hemoglobina $\mathrm{S}$ em testes de solubilidade. Revista Brasileira de Patologia Clínica $1977 ; 13: 133-34$

25. Cascudo LC. História da cidade do Natal. 2a Ed. Rio de Janeiro: Civilização Brasileira; 1980

26. Medeiros TA. Aspectos geopolíticos e antropológicos da história do Rio Grande do Norte. Natal: Imprensa Universitária; 1973.

27. Fundação Instituto Brasileiro de Geografia e Estatística. Resultados preliminares da amostra. http:// www.ibge.gov.br (acessado em 20/Nov/2002).

28. Ruiz MA, Guerra CCC, Naoum PC. Detecção de hemoglobinas anormais em sangue cordão de recém-nascidos na Cidade de Santos, São Paulo, através de eletroforese em gel de ágar de amido. Boletim da Sociedade Brasileira de Hematologia e Hemoterapia 1986; 8:8-13.

29. Serjeant BE, Forbes M, William LL, Serjeant GR. Screening cord blood for detection of cell disease in Jamaica. Clin Chem 1974; 20:666-9.

30. Charles C, Hopkinson E, Swan L, Morris E. Neonatal cord blood screening in Guyana: a preliminary report. West Indian Med J 1998; 47:58.

31. John MA, Lungu FM. Haemoglobin electrophoresis patterns in Barbados. West Indian M J 1999; 48:221-2.

32. Vichinsky E, Hurst D, Earles A, Kleman K, Lubin B. Newborn screening for sickle cell disease: effect on mortality. Pediatrics 1988; 81:749-55.

33. Ramalho AS, Jorge RN, Oliveira JA, Pereira DA. Hemoglobina "S" em recém-nascidos brasileiros. J Pediatr (Rio J.) 1976; 41:22-4.

34. Serjeant GR. Screening for sickle cell disease in Brasil. Lancet 2000; 356:168-9.

35. Portaria GM/MS no 822, de 06 de junho de 2001. Institui no âmbito do Sistema Único de Saúde o Programa Nacional de Triagem Neonatal/PNTN. Diário Oficial da União 2001; 7 jun

Recebido em 13/Jan/2003

Versão final reapresentada em 29/Mai/2003 Aprovado em 16/Set/2003 